\title{
RESEARCH
}

Open Access

\section{Arterial stiffening acts synergistically with APOE genotype and AD biomarker status to influence memory in older adults without dementia}

Katherine J. Bangen ${ }^{1,2^{*}}$ (D) Denis S. Smirnov ${ }^{3,4}$, Lisa Delano-Wood ${ }^{2,5}$, Christina E. Wierenga ${ }^{1,2}$, Mark W. Bondi ${ }^{2,5}$, David P. Salmon ${ }^{4}$ and Douglas Galasko ${ }^{4}$

\begin{abstract}
Background: Arterial stiffening has emerged as an important risk factor for Alzheimer's disease (AD) and related dementias. Carotid-femoral pulse wave velocity has been proposed as a non-invasive and reproducible method to assess arterial stiffness. However, the association of pulse wave velocity with performance across multiple cognitive domains as well as interactions with in vivo AD biomarkers and apolipoprotein E (APOE) genotype has received limited study.

Method: We studied 193 older adult volunteers (167 with normal cognition and 26 with mild cognitive impairment) who underwent comprehensive medical and neuropsychological evaluation at the University of California, San Diego Alzheimer's Disease Research Center. Cerebrospinal fluid (CSF) biomarkers were available on 123 participants (63\%). Linear models examined whether pulse wave velocity significantly interacted with APOE $\varepsilon 4$ status and CSF AD biomarker positivity (based on the ratio of total tau over beta-amyloid [tau/A $\left.\beta_{42}\right]$ ) on memory, language, executive functioning, attention, and visuospatial abilities.

Results: After adjusting for demographic characteristics and vascular risk burden, across the entire sample, pulse wave velocity was associated with poorer executive functioning but not the performance in the other cognitive domains. When the modifying effects of AD genetic risk and CSF AD biomarkers were considered, pulse wave velocity interacted with APOE genotype and CSF tau/A $\beta$ ratio such that a stronger association between elevated pulse wave velocity and poorer memory performance was found among those positive for CSF and genetic AD markers. There were no significant interaction effects for non-memory cognitive domains.
\end{abstract}

Conclusion: The findings suggest that pulse wave velocity, a non-invasive method to assess arterial wall properties, may be a useful marker of risk for cognitive decline, particularly among individuals who are APOE $\varepsilon 4$ carriers or CSF AD biomarke0r-positive.

Keywords: Alzheimer's disease, Pulse wave velocity, Arterial stiffening, Vascular risk factors, Cognition, Memory, Microcirculation, Aging

\footnotetext{
* Correspondence: kbangen@ucsd.edu

'Research Service, VA San Diego Healthcare System, Building 13, 3350 La Jolla Village Drive (151A), San Diego, CA 92161, USA

2Department of Psychiatry, University of California, San Diego, La Jolla, CA,

USA

Full list of author information is available at the end of the article
}

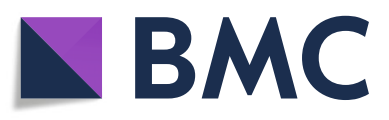

(c) The Author(s). 2021 Open Access This article is licensed under a Creative Commons Attribution 4.0 International License, which permits use, sharing, adaptation, distribution and reproduction in any medium or format, as long as you give appropriate credit to the original author(s) and the source, provide a link to the Creative Commons licence, and indicate if changes were made. The images or other third party material in this article are included in the article's Creative Commons licence, unless indicated otherwise in a credit line to the material. If material is not included in the article's Creative Commons licence and your intended use is not permitted by statutory regulation or exceeds the permitted use, you will need to obtain permission directly from the copyright holder. To view a copy of this licence, visit http://creativecommons.org/licenses/by/4.0/. The Creative Commons Public Domain Dedication waiver (http://creativecommons.org/publicdomain/zero/1.0/) applies to the data made available in this article, unless otherwise stated in a credit line to the data. 


\section{Introduction}

Risk factors for cerebrovascular disease can also increase the risk of developing Alzheimer's disease (AD) and are potentially modifiable to prevent or delay cognitive impairment [1,2]. Arterial stiffness underlies the effect of blood pressure on the brain and has emerged as an important non-traditional vascular risk factor [3, 4]. Arterial stiffening occurs with aging and is exacerbated by conditions such as hypertension and diabetes [5]. Pulse wave velocity (PWV) is a non-invasive and reproducible method to assess arterial stiffness [6] that has been linked to increased cerebral small vessel disease evidenced by white matter hyperintensities on magnetic resonance imaging (MRI) and to $\beta$-amyloid $(A \beta)$ accumulation associated with $\mathrm{AD}$ [1]. Indeed, increased PWV (i.e., greater arterial stiffness) has been proposed as a possible mechanism linking hypertension to both cerebral small vessel disease and $A \beta$ accumulation [1]. Increased PWV may damage the microcirculation due to elevated flow pulsatility $[7,8]$ and interfere with $A \beta$ clearance by impairing perivascular drainage [9].

Higher PWV has been shown to be associated with poorer cognitive performance (particularly episodic memory and executive functioning) $[7,10]$ in normally aging samples, mild cognitive impairment (MCI) [11], and cognitive decline over time $[12,13]$; however, findings have been inconsistent with some studies reporting the loss of this effect after adjustment for other cardiovascular risk factors [14]. The association between higher PWV and poorer cognition may be particularly salient in carriers of the apolipoprotein E (APOE) $\varepsilon 4$ allele [15], the strongest genetic risk factor for late-onset, sporadic AD [16, 17]. APOE $\varepsilon 4$ promotes blood-brain barrier (BBB) breakdown [18] and facilitates $A \beta$ accumulation in the brain parenchyma and blood vessel walls [19]. Notably, APOE ع4-mediated neurovascular defects appear to occur prior to neuronal dysfunction and degeneration [18], suggesting that APOE \&4 carriers may be more vulnerable than non-carriers to cerebrovascular dysfunction stemming from adverse vascular changes such as increased PWV [15].

Prior neuroimaging and neuropathological investigations suggest that the co-occurrence of cerebrovascular disease and AD-related changes lowers the threshold at which pathology is expressed as cognitive impairment $[20,21]$. These findings suggest that measures of arterial stiffening and in vivo measures of cerebrospinal fluid (CSF) tau and $A \beta$ may interact to reduce cognitive performance in older adults prior to the development of dementia. To test this possibility, we examined the relationships among PWV, genetic and CSF AD risk factors (APOE $\varepsilon 4$ genotype, CSF $A \beta_{42}$, and tau), and cognition in a well-characterized sample of older adults free of clinical dementia and stroke. We hypothesized that higher PWV would be associated with poorer episodic memory and executive functioning, especially in individuals at elevated risk of developing AD (i.e., APOE $\varepsilon 4$ carriers or CSF tau/A $\beta+$ ).

\section{Materials and methods \\ Standard protocol approvals and participant consents}

Participants for this cross-sectional study were selected from the ongoing longitudinal study of the University of California, San Diego (UCSD) Shiley-Marcos Alzheimer's Disease Research Center (ADRC) and ADRCaffiliated research studies. The research protocols were reviewed and approved by the human subject's review boards at UCSD and/or the VA San Diego Healthcare System. Informed consent was obtained from all participants.

\section{Participants}

Participants were 193 older adults without dementia who had completed clinical, neurological, and neuropsychological evaluations under the ongoing ADRC research study protocol [22, 23]. Non-demented participants are recruited to the ADRC from the community through community outreach events and wordof-mouth and from local practitioners who refer patients with possible memory deficits. The inclusion criteria for the present study included available PWV and neuropsychological test data and no current major physical/ medical health problems. The exclusion criteria included a history of major stroke, neurologic disorder, major psychiatric illness, substance abuse, or learning disability. Overall, the participants had a mean age of $74.9 \pm 5.9$ (mean \pm standard deviation) years and a mean of $16.7 \pm$ 2.4 years of formal education, and $53 \%$ were women and $10 \%$ were Hispanic. Based on the results of the ADRC evaluations, 167 participants were classified as cognitively normal $(\mathrm{CN})$ and 26 as $\mathrm{MCI}$ by consensus of a multidisciplinary team that included two senior neurologists and a neuropsychologist. Forty percent of the participants had at least one copy of the APOE $\varepsilon 4$ allele. This included 64/167 (38\%) CN participants and 14/26 (54\%) MCI participants. CSF was available for 123 participants $(64.7 \%$ of the sample; $103 \mathrm{CN}, 20 \mathrm{MCI})$. The use of anticoagulant medication was a contraindication for lumbar puncture (see Table 1 for participant characteristics across the entire sample; see supplemental tables comparing the characteristics of APOE $\varepsilon 4+$ versus APOE $\varepsilon 4-$ participants (Supplemental Table 1) and tau/ $\mathrm{A} \beta_{42}+$ versus tau $/ \mathrm{A} \beta_{42}-$ participants (Supplemental Table 2)). Notably, there were no significant group differences between APOE $\varepsilon 4+$ and APOE $\varepsilon 4-$ participants or between tau/A $\beta_{42}+$ and tau/A $\beta_{42}-$ participants, in terms of PWV. 
Table 1 Participant demographics and clinical characteristics

\begin{tabular}{|c|c|}
\hline Variable & Total sample, $N=193$, mean \pm SD or number $(\%)$ \\
\hline Age & $74.9 \pm 5.9$ \\
\hline Female & $102(53 \%)$ \\
\hline Education & $16.7 \pm 2.4$ \\
\hline APOE ع4+ & $78(40 \%)$ \\
\hline Hispanic & $18(10 \%)$ \\
\hline DRS & $138.9 \pm 4.3$ \\
\hline MMSE & $29.1 \pm 1.3$ \\
\hline CDR sum of boxes & $0.3 \pm 0.7$ \\
\hline GDS & $1.2 \pm 1.7$ \\
\hline Clinical Dx: normal & $167(87 \%)$ \\
\hline Clinical Dx: MCl & $26(13 \%)$ \\
\hline FSRP (\%) & $10.3 \pm 7.9$ \\
\hline Systolic BP & $131.7 \pm 18.6$ \\
\hline Diastolic BP & $80.4 \pm 11.2$ \\
\hline Pulse pressure & $51.2 \pm 13.9$ \\
\hline PWV & $8.9 \pm 2.1$ \\
\hline TIA & $3(2 \%)$ \\
\hline Atrial fibrillation & $20(10 \%)$ \\
\hline Diabetes & $12(6 \%)$ \\
\hline CVD & $27(14 \%)$ \\
\hline Smoking & $4(2 \%)$ \\
\hline Antihypertensive medication use & $94(49 \%)$ \\
\hline BMI & $26.0 \pm 5.6$ \\
\hline $\mathrm{N}$ with CSF measures & $123(64 \%)$ \\
\hline Interval from LP to PWV collection (years) & $1.2 \pm 0.9$ \\
\hline$A \beta_{42}$ & $812.2 \pm 378.6$ \\
\hline Tau & $358.8 \pm 190.1$ \\
\hline$A D$-like tau $/ A \beta_{42}$ ratio & $42(34 \%)$ \\
\hline
\end{tabular}

Abbreviations: CN cognitively normal, $M C I$ mild cognitive impairment, SD standard deviation, APOE apolipoprotein E, DRS Dementia Rating Scale, MMSE Mini-Mental State Examination, CDR Clinical Dementia Rating Scale, GDS Geriatric Depression Scale, FSRP Framingham Stroke Risk Profile, bp blood pressure. Pulse pressure was calculated as systolic blood pressure minus diastolic blood pressure. PWV pulse wave velocity, CVD cardiovascular disease, $B M I$ body mass index, CSF cerebrospinal fluid, Tau total tau; $A D$ Alzheimer's disease, $A \beta$ amyloid beta

\section{Neuropsychological evaluation}

The comprehensive neuropsychological test battery included measures of global cognitive function (Dementia Rating Scale, Mini Mental State Examination (MMSE), Clinical Dementia Rating (CDR)), memory (Wechsler Memory Scale-Revised (WMS-R) Visual Reproduction and Logical Memory, California Verbal List Learning Test (CVLT)), language (Multilingual Naming Test (MiNT), letter fluency (FAS), category fluency (animals, fruits, vegetables), Pyramids and Palm Trees), executive functions and attention (Modified Wisconsin Card Sorting Test, Color-Word Interference Test (CWIT), Trail Making Test Parts A and B, Wechsler Adult Scale of Intelligence-Revised (WAIS-R) Digit Symbol Substitution Test, WMS-R Digit Span), and visuospatial abilities
(Wechsler Intelligence Scale for Children-Revised (WISCR) Block Design Test, WMS-R Visual Reproduction copy).

Cognitive domain scores were generated through principal component analysis (PCA) with varimax rotation of all cognitive test scores (excluding global measures CDR, MMSE, and DRS) using previously described methods [24]. Prior to the PCA, a small percentage of missing scores for each test $(<5 \%$ for all tests, except for up to $10 \%$ for CWIT and visual reproduction copy) was imputed using a partial means matching approach based on demographics, diagnostic classification, and scores on global cognitive measures and the other cognitive tests. The PCA resulted in five components which were conceptually labeled "memory," "executive functioning," "language," "visuospatial abilities," and "attention" based 
on the tests with the highest loadings in each component (Table 2). A cognitive domain score was generated for each component based on the loadings for that component. Test scores from a pool of 146 "robust" normal controls (i.e., diagnosed as normal on their first ADRC evaluation and remained normal for the duration of their participation in the longitudinal study) were submitted to the same component loadings to generate "normal" cognitive domain scores that were used as reference values to "normalize" (i.e., convert cognitive domain scores to z-scores) the scores of the current study participants. The robust normal control participants were selected from the overall ADRC sample in a separate process not associated with the current study; however, 90 of the $146(62 \%)$ robust normal control participants were included in the present study.

\section{Vascular risk assessment: Framingham Stroke Risk Profile (FSRP)}

Estimated 10-year stroke risk (as a percentage) was derived using the revised Framingham Stroke Risk Profile (FSRP) [25] which was calculated using exponential equations with sex-specific baseline survival rates and sex-specific beta coefficients for the following risk factors: age, systolic blood pressure, diabetes mellitus, history of cardiovascular disease, atrial fibrillation, cigarette smoking, and use of antihypertensive medications.
Systolic blood pressure was the average of three blood pressure readings. Diabetes was defined as self-reported diabetes, use of an anti-diabetic therapy, or casual blood glucose $\geq 200 \mathrm{mg} / \mathrm{dL}$. History of cardiovascular disease (coronary artery disease [myocardial infarction, angina pectoris, coronary insufficiency], intermittent claudication, or cardiac failure) and atrial fibrillation were determined by clinical interview, physical exam, and review of outside medical care records and laboratory studies. Current smoking (yes/no) was based on self-report. The use of antihypertensive medications was ascertained through medication review. Height and weight were recorded, and body mass index (BMI) was calculated with the following formula: weight in kilograms/height in meters squared.

\section{Pulse wave velocity}

Aortic-femoral PWV was measured with the SphygmoCor XCEL device (AtCor Medical, Sydney, NSW, Australia) using well-validated procedures [26, 27] performed by trained operators. All measurements were collected with the participant in the supine position after $10 \mathrm{~min}$ of rest. Femoral pulse was measured using a blood pressure cuff around the upper thigh. Carotid pulse was simultaneously measured with a tonometer manually applied at the neck. On average, three PWV

Table 2 Principal component analysis factor loadings

\begin{tabular}{|c|c|c|c|c|c|}
\hline Measure & Memory & Executive & Language & Attention & Visuospatial \\
\hline Logical memory_immediate & 0.78 & 0.14 & 0.35 & 0.09 & 0.16 \\
\hline Logical memory_delayed & 0.82 & 0.14 & 0.30 & 0.10 & 0.16 \\
\hline CVLT-trials 1-5 & 0.85 & 0.28 & 0.11 & 0.14 & 0.09 \\
\hline CVLT_-short delay free & 0.88 & 0.28 & 0.07 & 0.10 & 0.06 \\
\hline CVLT-long delay free & 0.88 & 0.26 & 0.09 & 0.08 & 0.06 \\
\hline Visual reproduction_-immediate & 0.49 & 0.35 & 0.19 & 0.09 & 0.59 \\
\hline Visual reproduction-delay & 0.64 & 0.21 & 0.10 & 0.07 & 0.38 \\
\hline Trail A & -0.13 & -0.72 & -0.27 & -0.15 & -0.21 \\
\hline Trail B & -0.27 & -0.77 & -0.14 & -0.18 & -0.18 \\
\hline Digit symbol & 0.32 & 0.75 & 0.13 & 0.20 & 0.13 \\
\hline CWIT_inhibition & -0.22 & -0.76 & -0.12 & -0.08 & -0.05 \\
\hline WCST_categories & 0.30 & 0.48 & 0.16 & -0.01 & 0.31 \\
\hline Letter fluency & 0.25 & 0.5 & 0.37 & 0.42 & -0.21 \\
\hline Category fluency & 0.41 & 0.43 & 0.49 & 0.34 & -0.04 \\
\hline MiNT & 0.22 & 0.25 & 0.78 & 0.25 & 0.07 \\
\hline Pyramids and Palm Trees & 0.23 & 0.25 & 0.74 & -0.03 & 0.26 \\
\hline Visual reproduction-copy & 0.14 & 0.17 & 0.09 & 0.11 & 0.82 \\
\hline Block design & 0.19 & 0.60 & 0.36 & 0.20 & 0.40 \\
\hline Digit span-forward & 0.08 & 0.09 & 0.05 & 0.89 & 0.08 \\
\hline Digit span-backward & 0.16 & 0.34 & 0.20 & 0.70 & 0.18 \\
\hline
\end{tabular}

Abbreviations: CVLT California Verbal Learning Test, CWIT Color-Word Interference Test, WCST Wisconsin Card Sorting Test, MiNT Multilingual Naming Test 
measurements were recorded, and the mean value was used in statistical analyses.

\section{Biomarker acquisition and analysis}

Participants received a research lumbar puncture with standardized procedures, preanalytical preparation of CSF, and storage of CSF as previously described [28] and in accordance with the recommended best practices [29]. In brief, CSF $(15-25 \mathrm{~mL})$ was collected by routine lumbar puncture early in the morning after overnight fasting. Samples were processed, aliquoted into $500 \mu \mathrm{L}$ fractions in polypropylene microtubes, snap-frozen, and stored at $-80^{\circ} \mathrm{C}$ until assayed. Lumbar punctures were generally conducted within 1 year of the PWV measurements for the vast majority of the participants, although we included CSF data collected within 3 years of the baseline visit given research showing the stability of CSF biomarkers over several years [30].

CSF AD biomarkers examined were beta-amyloid (A 342$)$, total tau (Tau), and the ratio of tau over betaamyloid $\left(\operatorname{tau} / \mathrm{A} \beta_{42}\right)$. A small portion of cases $(n=18)$ had biomarkers analyzed in the facilities of ADx (Ghent, Belgium) using ELISA assays developed by ADx and commercialized by EUROIMMUN AG (Lubeck, Germany). The remaining cases' samples $(n=106)$ were analyzed locally at UCSD using the automated Lumipulse platform using assays developed with established monoclonal antibodies (Fujirebio Inc.) [31]. Weighted Deming regression of a set of 113 CSF samples analyzed on both platforms demonstrated high correlation (Pearson's R of .87 for $\mathrm{A} \beta 42$ and .95 for $\mathrm{Tau}$ ), so the resulting equations were used to transform ADx values into native Lumipulse space for analyses. A cutpoint for biomarker positivity in Lumipulse space was derived from CSF samples from a larger cohort of 462 unique participants at the UCSD ADRC (ranging from cognitively normal to severely demented). The ratio of tau/A $\beta_{42}$ was used as it appears to provide the best subdivision of individuals without dementia into those with and without pre-clinical AD [32]. A 2component mixture model was fit to the bimodal distribution of the log-transformed tau $/ A \beta_{42}$ ratio using the mixtools [33] package version 1.2.0 for $\mathrm{R}$, and the cutpoint of $\operatorname{tau} / \mathrm{A} \beta_{42}>0.52$ was chosen as the value at which an individual was equally likely to belong to either component of the bimodal distribution [34]. This cutpoint is highly consistent with a published cutpoint $\left(\operatorname{tau} / \mathrm{A} \beta_{42}>0.54\right)$ for $\mathrm{AD}$ biomarker positivity using the Lumipulse assays, derived against clinical read of amyloid PET scans and validated in multiple cohorts [31].

\section{Statistical analysis}

Demographics and clinical characteristics between those with and without CSF were compared by Student's t-test and chi-squared tests as appropriate. Relationships between PWV and performance in each cognitive domain were analyzed with separate linear models adjusted for age, sex, education, and FSRP. These analyses were then repeated with APOE genotype or CSF AD biomarker status $(+/-)$ added to explore the interaction of PWV and AD risk markers (APOE $\varepsilon 4+; \operatorname{tau} / \mathrm{A}_{42}+$ ) on cognition (while adjusting for age, sex, education, and FSRP).

Additional post hoc analyses were performed to determine whether significant results were retained in models including the individual FSRP components in place of the overall FSRP. That is, in cases where there were significant results, we reran the primary analyses described above but in place of overall FSRP instead adjusted for systolic blood pressure, history of diabetes, cardiovascular disease and/or atrial fibrillation, current smoking, and use of antihypertensive medication. We also reran these analyses with the individual FSRP components listed above while adjusting for two more vascular risk factors: BMI and history of transient ischemic attack (TIA).

\section{Results}

\section{Associations of PWV with participant characteristics}

Across the cohort, there was a significant increase in PWV with increasing age in both men and women $(p<$ 0.001 ), with no evidence of an interaction between sex and age $(p=.89)$. Overall, men had higher PWV than women $(p<0.001)$ (Fig. 1A). There was no significant association between PWV and APOE $\varepsilon 4$ status (Fig. 1B), nor between PWV and the level of CSF Tau or $A \beta_{42}$, or the tau/A $\beta_{42}$ ratio (Fig. 1C).

\section{Associations of PWV with cognitive test performance}

Performance on each of the cognitive domain scores is presented as a function of PWV in Fig. 2A. After adjusting for age, sex, education, and FSRP, PWV was significantly negatively associated with the executive domain score $(\beta=-0.09 \pm 0.04, p=.03)$, but not with the memory $(\beta=-0.03 \pm 0.04, p=.43)$, language $(\beta=0.06 \pm$ $0.04, p=.07)$, attention $(\beta=0.03 \pm 0.04, p=.42)$, or visuospatial abilities $(\beta=0.04 \pm 0.05, p=.31)$ domain scores (Fig. 2A).

\section{Interaction of APOE genotype with PWV on cognitive test performance}

Performance on each of the cognitive domain scores is presented as a function of PWV separately for APOE $\varepsilon 4+$ and $\varepsilon 4-$ participants in Fig. 2B. After adjusting for age, sex, education, and FSRP, there was a significant interaction between PWV and APOE genotype on the memory domain score (interaction $p=.01$ ) reflecting a larger decrease in performance with increasing PWV for the $\varepsilon 4+$ participants than for the $\varepsilon 4$ - participants. There 

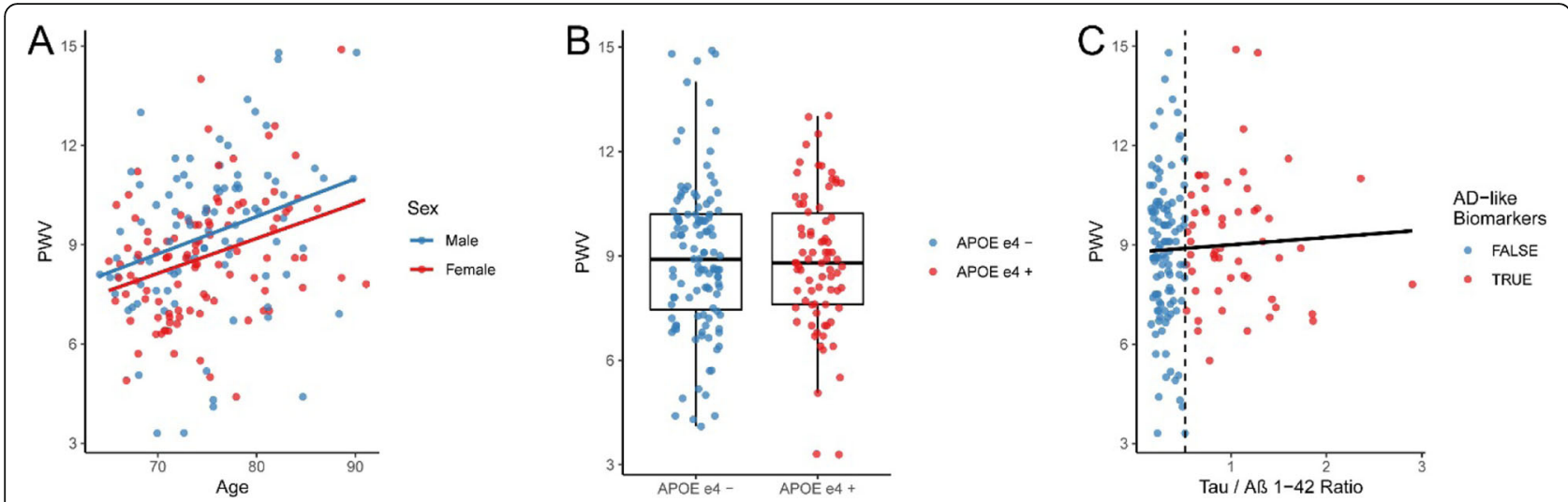

Fig. 1 PWV is associated with age and sex but does not differ by APOE $\varepsilon 4$ or AD CSF biomarker status. A PWV is shown as a function of age for male and female participants. Regression lines fit separately by sex show nearly identical slopes, with men showing consistently higher PWV across the age range. $\mathbf{B}$ Boxplot of PWV as a function of APOE genotype shows no difference between those with an APOE $\varepsilon 4$ allele ( $\varepsilon 2 \varepsilon 4$, $\varepsilon 3 \varepsilon 4$, $\varepsilon 4 \varepsilon 4)$ and those without ( $\varepsilon 2 \varepsilon 2, \varepsilon 2 \varepsilon 3, \varepsilon 3 \varepsilon 3)$. C PWV is plotted as a function of AD biomarker level (tau/A $\beta$ ratio). A regression line shows no association. The dashed vertical line represents the biomarker positivity threshold $(>0.52)$

was no interaction between PWV and APOE genotype for the executive functioning $(p=.73)$, language $(p=$ $.86)$, attention $(p=.13)$, or visuospatial abilities $(p=.21)$ domain scores (Fig. 2B).

\section{Interaction of AD biomarkers with PWV on cognitive test performance}

Performance on each of the cognitive domain scores is presented as a function of PWV separately for AD biomarker+ and $\mathrm{AD}$ biomarker- participants (defined by the tau $/ A \beta_{42}$ ratio cutoff) in Fig. 2C. After adjusting for age, sex, education, and FSRP, there was a significant interaction between PWV and AD biomarker positivity on the memory domain score (interaction $p=.01$ ) reflecting a larger decrease in performance with increasing PWV for the AD biomarker+ participants than for the AD biomarker- participants. There was no interaction between PWV and AD biomarker positivity for the executive functioning $(p=.69)$, language $(p=.97)$, attention $(p=.23)$, or visuospatial abilities $(p=.83)$ domain scores (Fig. 2C).

When additional post hoc analyses including individual vascular risk factors in place of overall FSRP score were performed, the results remained similar. That is, the significant main effect of the pulse wave velocity on executive function, and the significant interactions of pulse wave velocity and both APOE $\varepsilon 4$ status and CSF AD biomarker status on memory, remained significant (see Supplemental Tables 3, 4, and 5 for detailed results).

\section{Discussion}

We found that increased arterial stiffness measured through carotid-femoral PWV is associated with poorer performance on tests of executive functioning and memory in a well-characterized sample of older adults free of clinical dementia or stroke. The relationship between PWV and executive functioning was independent of the degree of AD risk as determined by APOE genotype or CSF AD biomarkers. In contrast, the relationship between elevated PWV and reduced memory performance was only present in at-risk participants by virtue of the APOE $\varepsilon 4$ genotype or an above threshold CSF tau/ $A \beta$ ratio. Notably, the observed relationships between PWV and cognition were apparent even after adjusting for important demographic factors and overall cardiovascular risk estimated by the FSRP.

Arterial stiffening increases with advancing age, particularly when cardiovascular disease risk factors are present [35]. This increase in arterial stiffening results in higher PWV and pressure pulsatility, a reduction of the normal protective gradient of increasing stiffness moving from aorta to periphery, transmission of potentially harmful pulsatile energy, and reactive changes to microvascular function and structure that may compromise blood flow [8]. High-flow organs such as the brain may be particularly vulnerable to these secondary changes to the microcirculation and reduced perfusion [8]. Consistent with this possibility, we found that elevated PWV, a reflection of increased arterial stiffness, was associated with poorer executive function regardless of the degree of $\mathrm{AD}$ risk. Executive functioning has been repeatedly shown to decline in the context of normal aging [36], and indeed, "normal" cognitive aging has been conceptualized as a selective vulnerability in frontal-subcortical executive processes [37, 38]. Our results suggest that arterial stiffening may contribute to this age-related decline (see [39]). Interestingly, however, we observed a relationship between PWV and executive functioning even after adjusting for age. This suggests that elevated 

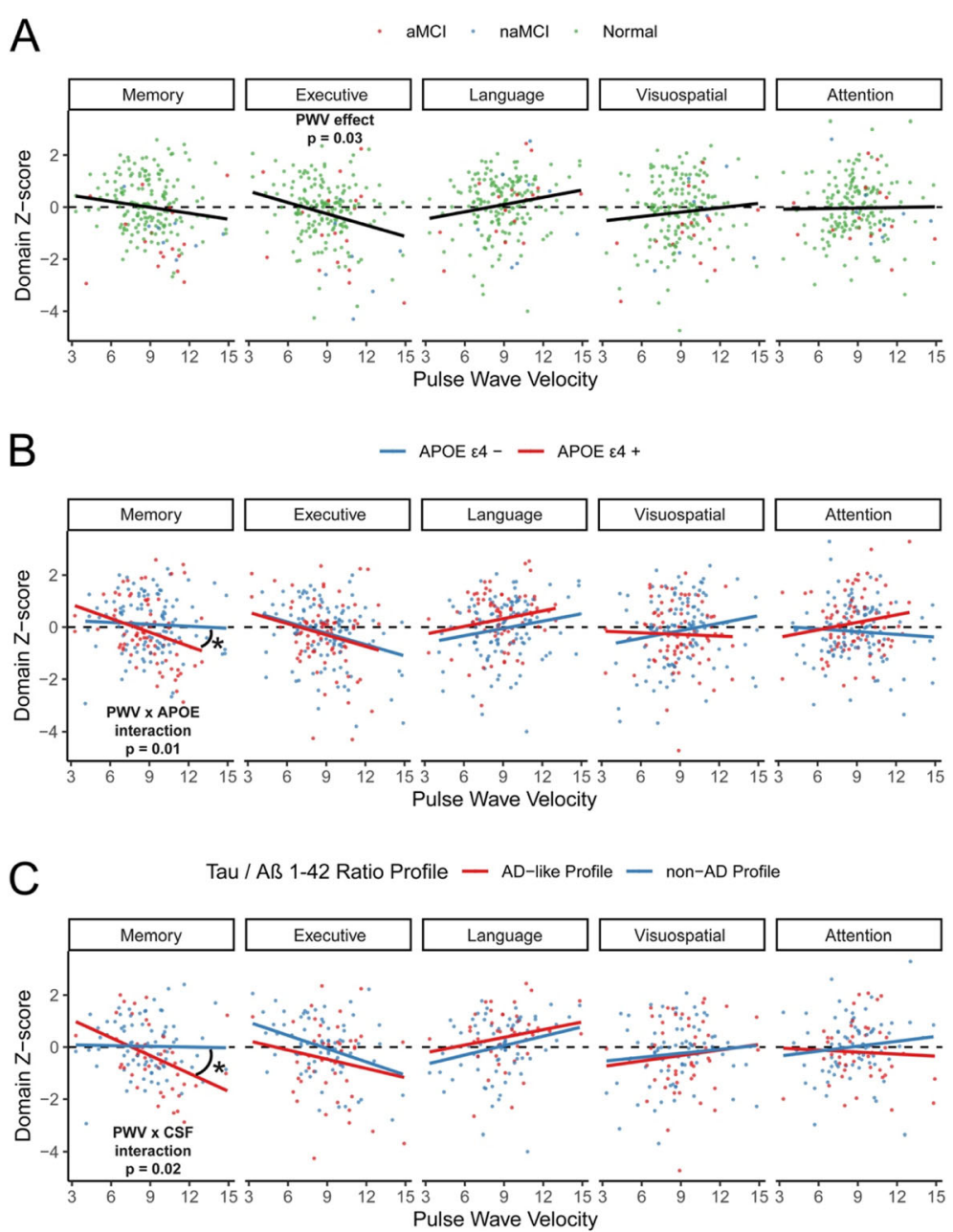

Fig. 2 PWV predicts executive function beyond age and sex and interacts with APOE genotype and AD CSF biomarkers to predict memory performance. A Cognitive domain z-scores are shown as a function of PWV. Regression models adjusted for age, sex, education, and FSRP (excluding age points) demonstrate a significant main effect of PWV on executive function $(p=0.03)$. B The same cases were classified based on APOE genotype as APOE $\varepsilon 4+(\varepsilon 2 \varepsilon 4, \varepsilon 3 \varepsilon 4, \varepsilon 4 \varepsilon 4)$ or APOE $\varepsilon 4-(\varepsilon 2 \varepsilon 2, \varepsilon 2 \varepsilon 3, \varepsilon 3 \varepsilon 3)$. In regression models examining the interaction of PWV and APOE genotype, a significant interaction ( $p=0.01$ ) suggested that PWV was more predictive of memory scores in those that were APOE $\varepsilon 4+$. C In a subset of patients with CSF biomarkers, individuals were classified into AD-like and non-AD profiles based on the tau/A $\beta_{1-42}$ ratio. In regression models examining the interaction of PWV and CSF AD biomarker status, a significant interaction $(p=0.02)$ suggests that PWV was similarly more predictive of memory z-scores in those that were AD-like in their profile

PWV may relate to executive decline over and beyond normal, expected age-related changes [39], and this finding is consistent with prior evidence demonstrating that vascular damage preferentially affects frontally mediated executive abilities [40, 41].

Our finding that elevated PWV interacts with APOE genotype and CSF tau/A $\beta_{42}$ ratio to influence memory function is consistent with previous research suggesting that high pulse pressure and vascular risk factors may interact with genetic risk for $\mathrm{AD}$ and markers of $\mathrm{AD}$ related pathology to confer an increased risk of cognitive decline [39, 42, 43]. For example, a previous study found that APOE \&4 carriers demonstrated poorer cognitive performance than non-carriers among participants with higher aortic PWV [15]. The authors proposed that APOE $\varepsilon 4$ carriers may be more vulnerable to vascular changes given a loss or reduction of normal protective mechanisms that may be compromised due to existing neurovascular dysfunction and increasing neuropathology. It is possible that there is a threshold effect such that the adverse impact of higher PWV on cognition in at-risk adults may emerge at PWV values that are not detrimental to cognition in older adults with low risk of $\mathrm{AD}[15]$.

Our results are also consistent with previous research that has shown reduced regional cerebral blood flow and increased regional cerebrovascular resistance in older adults with elevated vascular risk and in those with $\mathrm{MCI}$ 
and AD [44-46]. These cerebrovascular changes were observed in the subcortical, frontal, and medial temporal regions, and they were linked to poorer cognitive abilities. Impaired cerebrovascular regulation and cerebral hypoperfusion may be most harmful in these brain regions because they are among the most metabolically active, are supplied by terminal arteries, and thereby are susceptible to the effects of small vessel disease, and may be affected by $A \beta$-mediated vasoconstriction $[15,46]$. Our finding of associations between elevated PWV and reduced memory performance in those with $\mathrm{AD}$ risk (i.e., APOE e4+ or AD CSF biomarker+) dovetail with this prior work and may reflect the vulnerability of the frontal and hippocampal regions to vascular dysfunction and accumulating ischemic insults $[47,48]$.

The present results contribute to a growing body of research that suggests vascular vulnerabilities interact with $\mathrm{AD}$ pathophysiology to increase the risk of neurodegeneration [39, 43] and cognitive decline [39, 42]. We and others have previously shown that elevated vascular risk burden influences the clinical expression of AD [21] whereby the overlap of cerebrovascular disease and $A \beta$ deposition in the brain lowers the threshold at which an individual with accumulating neuropathology develops cognitive impairment $[21,49]$. The two-hit hypothesis of AD proposed by Zlokovic states that vascular disease may contribute to $\mathrm{AD}$ pathophysiology through bloodbrain barrier breakdown resulting in the leakage of neurotoxic molecules into the tissue and/or through chronic hypoperfusion [2]. It also has been suggested that cerebrovascular abnormalities may impair $A \beta$ clearance via impairment of perivascular drainage $[9,50]$ and may also directly lead to neurodegeneration independent of cerebral amyloidosis [4]. Indeed, greater arterial stiffening has been associated with the presence of multiple forms of neuropathology including white matter disease, cerebral microbleeds, and tau and $A \beta$ deposition $[1,4]$. Given that vascular risk factors such as arterial stiffening are potentially modifiable, their association with cognition suggests that in some cases, cognitive impairment and dementia may be prevented and/or delayed [51]. Targeting arterial stiffening and other vascular risk factors through lifestyle and/or pharmacological interventions may be a promising way to facilitate brain health in aging.

Although PWV has been associated with white matter hyperintensity volume (WMH) [1], studies suggests that WMH only partially mediates the effect of hypertension on cognitive performance [52]. A recent study, for example, showed that microvascular dysfunction measured as a composite of MRI WMH volume and biomarkers of microvascular disease (e.g., plasma soluble intercellular adhesion molecule-1 [sICAM-1] and soluble vascular adhesion molecule-1 [sVCAM-1]) explained only $16.2 \%$ of the effect of aortic stiffness on cognitive function [53]. Studies examining WMH volume and other vascular risk factors such as diabetes also have not found an imaging metric that fully explains the association between the risk factor and cognitive performance $[54,55]$. Taken together, these studies highlight the importance of examining vascular risk factors in and of themselves rather than only the downstream end-organ damage they may cause [55].

The strengths of this study include the use of a wellvalidated carotid-femoral PWV measurement to assess arterial stiffening, administration of a comprehensive neuropsychological test battery that allowed empirical derivation of cognitive domain scores, and examination of CSF tau and $A \beta$ biomarkers to detect AD pathology in non-demented older adults. In addition, we adjusted for important demographic and vascular risk factor variables that influence cognition including age, sex, and aggregate vascular risk (as well as the individual FSRP components, BMI, and history of TIA in post hoc analyses), and our findings remained statistically significant suggesting PWV is an independent contributor to cognitive performance.

\section{Limitations}

Our study is limited in generalizability given the cohort is a relatively homogeneous sample of older adults who were predominantly White and well-educated with relatively good vascular health. Indeed, the prevalence of FSRP components of cardiovascular disease (14\%) and diabetes $(6 \%)$ in our sample were lower than in the general population over age 65 (24 to $37 \%$ for cardiovascular disease and 22 to $23 \%$ for diabetes) [56]. It is likely that associations between PWV and cognitive abilities would have been stronger in a sample with a greater vascular risk burden, and these associations should be explored in people with more prominent risk factors in future studies. Nonetheless, our findings suggest that even relatively mild vascular dysfunction may contribute to poorer cognitive function in individuals with elevated $\mathrm{AD}$ risk. In addition, the percentage of APOE $\varepsilon 4+$ cognitively normal participants in our cohort (38\%) is higher than reported in some previously published studies. AD case-control studies combining data from several research groups reported that among Caucasian individuals, approximately $14 \%$ of cognitively normal controls were APOE $\varepsilon 4$ carriers $[57,58]$ (although allele frequencies are known to vary based on factors such as sex, ethnicity, and geography $[59,60])$. However, the percentage of APOE $\varepsilon 4+$ cognitively normal participants in our cohort is very similar to those reported in other AD cohort studies (e.g., Uniform Data Set of the Alzheimer's Disease Centers [UDS] program and Australian Imaging, Biomarkers and Lifestyle Flagship Study of Ageing 
[AIBL]) [61]. Notably, there is bias in recruitment for our study and other $\mathrm{AD}$ cohort studies that results in enrichment for APOE $\varepsilon 4$ carriers, and this limits the generalizability of our findings to the general population [61]. We also do not have available FLAIR WMH data for our cohort, which will be critical to include in future studies to better characterize the presence and extent of vascular disease in our participants. Future longitudinal studies are necessary to determine if APOE genotype and AD biomarkers influence the trajectories of PWV and its relationship to cognitive decline as well as to explore some of the potential mechanisms underlying these relationships.

\section{Conclusions}

The findings of the current study showed that elevated PWV is associated with poorer cognitive performance in older adults, particularly on memory tasks, in those who are at risk for $\mathrm{AD}$. These findings suggest that PWV, a non-invasive method to assess arterial stiffness, should be explored as a potential marker of risk for cognitive decline in older adults. PWV appears to have added predictive value beyond the FSRP and individual FSRP variables, including blood pressure. While previous studies suggest that PWV correlates strongly with fluidattenuated inversion recovery (FLAIR) MRI WMH measures [1], PWV is much easier and less expensive to acquire than MRI and can be measured and tracked in a clinician's primary care office. In addition, there are very few contraindications of PWV assessment, whereas many older adults have contraindications to MRI (e.g., pacemakers, metal implants, claustrophobia). Our results also provide a rationale for attempts to improve central vascular function and decrease cerebral pulsatility as an intervention to reduce dementia risk by preventing or slowing the accumulation of $\mathrm{AD}$ and vascular neuropathology across the aging spectrum $[1,39]$.

\footnotetext{
Abbreviations

AD: Alzheimer's disease; APOE: Apolipoprotein E; CSF: Cerebrospinal fluid; Aß: Beta-amyloid; PWV: Pulse wave velocity; MCl: Mild cognitive impairment; BBB: Blood-brain barrier; UCSD: University of California, San Diego; ADRC: Alzheimer's Disease Research Center; CN: Cognitively normal; MMSE: Mini-Mental State Examination; CDR: Clinical Dementia Rating; WMSR: Wechsler Memory Scale-Revised; CVLT: California Verbal List Learning Test; MiNT: Multilingual Naming Test; CWIT: Color-Word Interference Test; WAISR: Wechsler Adult Scale of Intelligence-Revised; WISC-R: Wechsler Intelligence Scale for Children-Revised; PCA: Principal component analysis; FSRP: Framingham Stroke Risk Profile; BMI: Body mass index; TIA: Transient ischemic attack
}

\section{Supplementary Information}

The online version contains supplementary material available at https://doi. org/10.1186/s13195-021-00851-2.

Additional file 1: Table S1. Participant Demographics and Clinical Characteristics by APOE $\varepsilon 4$ Status. Table S2. Participant Demographics and Clinical Characteristics by AD CSF Biomarker Status. Table S3. Posthoc Exploration of Influence of Individual Vascular Risk Variables in Place of Overall Framingham Stroke Risk Profile: Results of Models Examining Main Effect of PW on Executive Function. Table S4. Post-hoc Exploration of Influence of Individual Vascular Risk Variables in Place of Overall Framingham Stroke Risk Profile: Results of Models Examining Interaction of PWV and APOE \&4 Status on Memory. Table S5. Post-hoc Exploration of Influence of Individual Vascular Risk Variables in Place of Overall Framingham Stroke Risk Profile: Results of Models Examining Interaction of PWV and CSF AD Biomarker Status on Memory.

\section{Acknowledgements}

The authors would like to thank the dedicated UCSD ADRC study coordinators and staff members who contributed to the collection of the pulse wave velocity data.

\section{Authors' contributions}

K.J.B., D.S.S., D.P.S., and D.G. designed the study. K.J.B. and D.S.S. analyzed the data. D.S.S. made the figures. K.J.B., D.S.S., L.D-W., C.E.W., M.W.D., D.P.S., and

D.G. drafted and revised the paper. All authors read and approved the final manuscript.

\section{Funding}

This work was supported by the US Department of Veterans Affairs Clinical Sciences Research and Development Service (Merit Award 1I01CX001842 to K.J.B., Merit Award 1I01CX000565 to C.E.W.), NIA/NIH grants (NIH P30 AG062429 to D.G. and D.P.S.; R01 AG063782 to K.J.B.; R01 AG049810 and R01 AG054049 to M.W.B.), and the Alzheimer's Association (AARG-18-566254 to K.J.B).

\section{Availability of data and materials}

Anonymized datasets analyzed in the current study are available to qualified investigators on reasonable request.

\section{Declarations}

Ethics approval and consent to participate

All procedures were approved by the Institutional Review Boards at UCSD. Written informed consent was obtained from every research participant according to the Declaration of Helsinki and the Belmont Report.

\section{Consent for publication}

Not applicable.

\section{Competing interests}

D. Galasko serves as an editor for Alzheimer's Research and Therapy; is a consultant for Biogen, Inc., Fujirebio, Inc., and Amprion, Inc.; and serves on a Data Safety Monitoring Board for Cognition Therapeutics. D.P. Salmon serves as a paid consultant for Takeda Pharmaceuticals, Inc.; Aptinyx, Inc.; and Biogen, Inc. M. Bondi receives royalties from Oxford University Press. Other authors declare that they have no competing interests.

\section{Author details}

${ }^{1}$ Research Service, VA San Diego Healthcare System, Building 13, 3350 La Jolla Village Drive (151A), San Diego, CA 92161, USA. ²Department of Psychiatry, University of California, San Diego, La Jolla, CA, USA. ${ }^{3}$ Medical Scientist Training Program, University of California, San Diego, La Jolla, CA, USA. ${ }^{4}$ Department of Neurosciences, University of California, San Diego, La Jolla, CA, USA. ${ }^{5}$ Psychology Service, VA San Diego Healthcare System, San Diego, CA, USA.

Received: 4 January 2021 Accepted: 31 May 2021 Published online: 01 July 2021

\section{References}

1. Hughes TM, Wagenknecht LE, Craft S, et al. Arterial stiffness and dementia pathology: Atherosclerosis Risk in Communities (ARIC)-PET Study. Neurology. 2018:90:e1248-56. 2018/03/20. https://doi.org/10.1212/wnl.00000000000052 59. 
2. Zlokovic BV. Neurovascular pathways to neurodegeneration in Alzheimer's disease and other disorders. Nat Rev Neurosci. 2011;12:723-38. 2011/11/04. https://doi.org/10.1038/nrn3114.

3. Hughes TM, Craft S, Lopez OL. Review of 'the potential role of arterial stiffness in the pathogenesis of Alzheimer's disease'. Neurodegener Dis Manag. 2015;5:121-35. 2015/04/22. https://doi.org/10.2217/nmt.14.53.

4. Nation DA, Edmonds EC, Bangen K, et al. Pulse pressure in relation to taumediated neurodegeneration, cerebral amyloidosis, and progression to dementia in very old adults. JAMA Neurol. 2015;72:546-53. 2015/03/31. https://doi.org/10.1001/jamaneurol.2014.4477.

5. Cockcroft JR, Webb DJ, Wilkinson IB. Arterial stiffness, hypertension and diabetes mellitus. J Hum Hypertens. 2000;14:377-80. 2000/07/06. https://doi. org/10.1038/sj.jhh.1001023.

6. Rabkin SW. Arterial stiffness: detection and consequences in cognitive impairment and dementia of the elderly. J Alzheimers Di. 2012;32:541-9. 2012/08/14. https://doi.org/10.3233/jad-2012-120757.

7. Mitchell GF, van Buchem MA, Sigurdsson S, et al. Arterial stiffness, pressure and flow pulsatility and brain structure and function: the Age, Gene/ Environment Susceptibility--Reykjavik Study. Brain. 2011;134:3398-407. 2011/ 11/15. https://doi.org/10.1093/brain/awr253.

8. Mitchell GF. Effects of central arterial aging on the structure and function of the peripheral vasculature: implications for end-organ damage. J Appl Physiol (Bethesda, MD: 1985). 2008;105:1652-60. 2008/09/06. https://doi. org/10.1152/japplphysiol.90549.2008.

9. Weller RO, Boche D, Nicoll JA. Microvasculature changes and cerebral amyloid angiopathy in Alzheimer's disease and their potential impact on therapy. Acta Neuropathol. 2009;118:87-102. 2009/02/24. https://doi.org/1 0.1007/s00401-009-0498-z.

10. Zhong W, Cruickshanks KJ, Schubert CR, et al. Pulse wave velocity and cognitive function in older adults. Alzheimer Dis Assoc Disord. 2014;28:44-9. 2013/05/02. https://doi.org/10.1097/WAD.0b013e3182949f06.

11. Meyer ML, Palta P, Tanaka H, et al. Association of central arterial stiffness and pressure pulsatility with mild cognitive impairment and dementia: the Atherosclerosis Risk in Communities Study-Neurocognitive Study (ARICNCS). J Alzheimers Dis. 2017;57:195-204. 2017/02/23. https://doi.org/1 $0.3233 /$ jad-161041

12. Watson NL, Sutton-Tyrrell K, Rosano C, et al. Arterial stiffness and cognitive decline in well-functioning older adults. J Gerontol A Biol Sci Med Sci. 2011; 66:1336-42. 2011/07/20. https://doi.org/10.1093/gerona/glr119.

13. Waldstein SR, Rice SC, Thayer JF, et al. Pulse pressure and pulse wave velocity are related to cognitive decline in the Baltimore Longitudinal Study of Aging. Hypertension. 2008;51:99-104. 2007/11/21. https://doi.org/10.11 61/hypertensionaha.107.093674.

14. Poels MM, van Oijen M, Mattace-Raso FU, et al. Arterial stiffness, cognitive decline, and risk of dementia: the Rotterdam Study. Stroke. 2007;38:888-92. 2007/02/03. https://doi.org/10.1161/01.Str.0000257998.33768.87.

15. Cambronero FE, Liu D, Neal JE, et al. APOE genotype modifies the association between central arterial stiffening and cognition in older adults. Neurobiol Aging. 2018;67:120-7. 2018/04/16. https://doi.org/10.1016/j. neurobiolaging.2018.02.009.

16. Corder EH, Saunders AM, Strittmatter WJ, et al. Gene dose of apolipoprotein E type 4 allele and the risk of Alzheimer's disease in late onset families. Science. 1993:261:921-3 1993/08/13.

17. Saunders NB, Zollinger WD, Rao VB. A rapid and sensitive PCR strategy employed for amplification and sequencing of porA from a single colonyforming unit of Neisseria meningitidis. Gene. 1993;137:153-62 1993/12/31.

18. Bell RD, Winkler EA, Singh I, et al. Apolipoprotein E controls cerebrovascular integrity via cyclophilin A. Nature. 2012;485:512-6. 2012/05/25. https://doi. org/10.1038/nature11087.

19. Castellano JM, Kim J, Stewart FR, et al. Human apoE isoforms differentially regulate brain amyloid- $\beta$ peptide clearance. Sci Transl Med. 2011;3:89ra57. 2011/07/01. https://doi.org/10.1126/scitranslmed.3002156.

20. Provenzano FA, Muraskin J, Tosto G, et al. White matter hyperintensities and cerebral amyloidosis: necessary and sufficient for clinical expression of Alzheimer disease? JAMA Neurol. 2013;70:455-61. 2013/02/20. https://doi. org/10.1001/jamaneurol.2013.1321.

21. Bangen KJ, Nation DA, Delano-Wood L, et al. Aggregate effects of vascular risk factors on cerebrovascular changes in autopsy-confirmed Alzheimer's disease. Alzheimers Dement J Alzheimers Assoc. 2015;11:394-403.e391. 2014/07/16. https://doi.org/10.1016/j.jalz.2013.12.025.
22. Galasko D, Hansen LA, Katzman R, et al. Clinical-neuropathological correlations in Alzheimer's disease and related dementias. Arch Neurol. 1994;51:888-95. 1994/09/01. https://doi.org/10.1001/archneur.1994.0054021 0060013.

23. Salmon DP, Butters N. Neuropsychological assessment of dementia in the elderly. In: Katzman R, Rowe JW, editors. Principles of Geriatric Neurology. Philadelphia: F.A. Davis; 1992. p. 144-63.

24. Smirnov DS, Galasko D, Edland SD, et al. Cognitive decline profiles differ in Parkinson disease dementia and dementia with Lewy bodies. Neurology. 2020;94:e2076-87. 2020/04/26. https://doi.org/10.1212/wnl. 0000000000009434

25. Dufouil C, Beiser A, McLure LA, et al. Revised Framingham Stroke Risk Profile to Reflect Temporal Trends. Circulation. 2017;135:1145-59. 2017/02/06. https://doi.org/10.1161/circulationaha.115.021275.

26. Laurent S, Cockcroft J, Van Bortel L, et al. Expert consensus document on arterial stiffness: methodological issues and clinical applications. Eur Heart J. 2006;27:2588-605. 2006/09/27. https://doi.org/10.1093/eurheartj/ehl254.

27. Hwang MH, Yoo JK, Kim HK, et al. Validity and reliability of aortic pulse wave velocity and augmentation index determined by the new cuff-based SphygmoCor Xcel. J Hum Hypertens. 2014;28:475-81. 2014/01/17. https:// doi.org/10.1038/jhh.2013.144

28. Xiao MF, Xu D, Craig MT, et al. NPTX2 and cognitive dysfunction in Alzheimer's disease. Elife. 2017;6 2017/04/26. https://doi.org/10.7554/eLife.23 798.

29. Vanderstichele H, Bibl M, Engelborghs S, et al. Standardization of preanalytical aspects of cerebrospinal fluid biomarker testing for Alzheimer's disease diagnosis: a consensus paper from the Alzheimer's Biomarkers Standardization Initiative. Alzheimers Dement J Alzheimers Assoc. 2012:8: 65-73. 2011/11/04. https://doi.org/10.1016/j.jalz.2011.07.004.

30. Lleó A, Alcolea D, Martínez-Lage P, et al. Longitudinal cerebrospinal fluid biomarker trajectories along the Alzheimer's disease continuum in the BIOMARKAPD study. Alzheimers Dement J Alzheimers Assoc. 2019:15:74253. 2019/04/11. https://doi.org/10.1016/j.jalz.2019.01.015.

31. Kaplow J, Vandijck M, Gray J, et al. Concordance of Lumipulse cerebrospinal fluid t-tau/Aß42 ratio with amyloid PET status. Alzheimers Dement J Alzheimers Assoc. 2020;16:144-52. 2020/01/09. https://doi.org/10.1002/alz.12000.

32. Van Harten AC, Wiste HJ, Weigand SD, et al. CSF biomarkers in Olmsted County: evidence of 2 subclasses and associations with demographics. Neurology. 2020;95:e256-67. 2020/06/28. https://doi.org/10.1212/wnl. 0000000000009874

33. Benaglia T, Chauveau D, Hunter DR, et al. mixtools: an $R$ package for analyzing mixture models. J Stat Softw. 2009:32:29. 2009-10-14. https://doi. org/10.18637/jss.v032.106.

34. Bartlett JW, Frost C, Mattsson N, et al. Determining cut-points for Alzheimer's disease biomarkers: statistical issues, methods and challenges. Biomark Med. 2012;6:391-400. 2012/08/25. https://doi.org/10.2217/bmm.12.49.

35. Mitchell GF, Guo CY, Benjamin EJ, et al. Cross-sectional correlates of increased aortic stiffness in the community: the Framingham Heart Study. Circulation. 2007;115:2628-36. 2007/05/09. https://doi.org/10.1161/circula tionaha.106.667733.

36. Hultsch DF, Hertzog C, Small BJ, et al. Short-term longitudinal change in cognitive performance in later life. Psychol Aging. 1992;7:571-84. 1992/12/ 01. https://doi.org/10.1037//0882-7974.7.4.571.

37. Daigneault S, Braun CM. Working memory and the self-ordered pointing task: further evidence of early prefrontal decline in normal aging. J Clin Exp Neuropsychol. 1993;15:881-95. 1993/11/01. https://doi.org/10.1080/0168863 9308402605.

38. McPherson S, Koltai D. A practical guide to geriatric neuropsychology. New York: Oxford University Press; 2018

39. Nation DA, Preis SR, Beiser A, et al. Pulse pressure is associated with early brain atrophy and cognitive decline: modifying effects of APOE-epsilon4. Alzheimer Dis Assoc Disord. 2016;30:210-5. 2016/08/25. https://doi.org/10.1 097/wad.0000000000000127.

40. Lamar M, Price CC, Giovannetti T, et al. The dysexecutive syndrome associated with ischaemic vascular disease and related subcortical neuropathology: a Boston process approach. Behav Neurol. 2010;22:53-62. 2009/01/01. https://doi.org/10.3233/ben-2009-0237.

41. Libon DJ, Drabick DA, Giovannetti T, et al. Neuropsychological syndromes associated with Alzheimer's/vascular dementia: a latent class analysis. J Alzheimers Dis. 2014:42:999-1014. 2014/07/16. https://doi.org/10.3233/jad-132147. 
42. Bangen KJ, Beiser A, Delano-Wood L, et al. APOE genotype modifies the relationship between midlife vascular risk factors and later cognitive decline. J Stroke Cerebrovasc Dis. 2013;22:1361-9. 2013/04/23. https://doi.org/10.101 6/j.jstrokecerebrovasdis.2013.03.013.

43. Bangen KJ, Himali JJ, Beiser AS, et al. Interaction between midlife blood glucose and APOE genotype predicts later Alzheimer's disease pathology. J Alzheimers Dis. 2016;53:1553-62. 2016/07/10. https://doi.org/10.3233/JAD-1 60163.

44. Bangen KJ, Werhane ML, Weigand AJ, et al. Reduced regional cerebral blood flow relates to poorer cognition in older adults with type 2 diabetes. Front Aging Neurosci. 2018;10. Original Research. https://doi.org/10.3389/fna gi.2018.00270

45. Clark LR, Nation DA, Wierenga $C E$, et al. Elevated cerebrovascular resistance index is associated with cognitive dysfunction in the very-old. Alzheimers Res Ther. 2015;7:3. 2015/01/01. https://doi.org/10.1186/s13195-014-0080-3.

46. Nation DA, Wierenga CE, Clark LR, et al. Cortical and subcortical cerebrovascular resistance index in mild cognitive impairment and Alzheimer's disease. J Alzheimers Dis. 2013;36:689-98. 2013/05/15. https:// doi.org/10.3233/jad-130086.

47. Raz N, Rodrigue KM, Acker JD. Hypertension and the brain: vulnerability of the prefrontal regions and executive functions. Behav Neurosci. 2003;117: 1169-80. 2003/12/17. https://doi.org/10.1037/0735-7044.117.6.1169.

48. Zarow C, Vinters HV, Ellis WG, et al. Correlates of hippocampal neuron number in Alzheimer's disease and ischemic vascular dementia. Ann Neurol. 2005;57:896-903. 2005/06/02. https://doi.org/10.1002/ana.20503.

49. Arvanitakis Z, Capuano AW, Leurgans SE, et al. Relation of cerebral vessel disease to Alzheimer's disease dementia and cognitive function in elderly people: a cross-sectional study. Lancet Neurol. 2016;15:934-43. 2016/06/18. https://doi.org/10.1016/s1474-4422(16)30029-1.

50. Weller RO, Subash M, Preston SD, et al. Perivascular drainage of amyloidbeta peptides from the brain and its failure in cerebral amyloid angiopathy and Alzheimer's disease. Brain Pathol. 2008;18:253-66. 2008/03/28. https:// doi.org/10.1111/j.1750-3639.2008.00133.x.

51. Gottesman RF, Albert MS, Alonso A, et al. Associations between midlife vascular risk factors and 25-year incident dementia in the Atherosclerosis Risk in Communities (ARIC) cohort. JAMA Neurol. 2017;74:1246-54. 2017/08/ 08. https://doi.org/10.1001/jamaneurol.2017.1658.

52. Hajjar I, Quach L, Yang F, et al. Hypertension, white matter hyperintensities, and concurrent impairments in mobility, cognition, and mood: the Cardiovascular Health Study. Circulation. 2011;123:858-65. 2011/02/16. https://doi.org/10.1161/circulationaha.110.978114.

53. Rensma SP, Stehouwer CDA, Van Boxtel MPJ, et al. Associations of arterial stiffness with cognitive performance, and the role of microvascular dysfunction: the Maastricht Study. Hypertension. 2020;75:1607-14. 2020/04/ 11. https://doi.org/10.1161/hypertensionaha.119.14307.

54. Callisaya ML, Beare R, Moran C, et al. Type 2 diabetes mellitus, brain atrophy and cognitive decline in older people: a longitudinal study. Diabetologia. 2019;62:448-58. 2018/12/14. https://doi.org/10.1007/s00125-018-4778-9.

55. Zlokovic BV, Gottesman RF, Bernstein KE, et al. Vascular contributions to cognitive impairment and dementia (VCID): a report from the 2018 National Heart, Lung, and Blood Institute and National Institute of Neurological Disorders and Stroke Workshop. Alzheimers Dement J Alzheimers Assoc. 2020;16:1714-33. 2020/10/09. https://doi.org/10.1002/alz.12157.

56. Villarroel MA, Blackwell DL, Jen A. Tables of summary health statistics for $U$. S. adults: 2018 National Health Interview Survey. National Center for Health Statistics; 2019. Available from: http://www.cdc.gov/nchs/nhis/SHS/tables.htm.

57. Farrer $L A$, Cupples $L A$, Haines $J$, et al. Effects of age, sex, and ethnicity on the association between apolipoprotein E genotype and Alzheimer disease. A meta-analysis. APOE and Alzheimer Disease Meta Analysis Consortium. JAMA. 1997;278:1349-56 1997/10/29.

58. Bang OY, Kwak YT, Joo IS, et al. Important link between dementia subtype and apolipoprotein E: a meta-analysis. Yonsei Med J. 2003;44:401-13. 2003/ 07/02. https://doi.org/10.3349/ymj.2003.44.3.401.

59. Zannis VI, Kardassis D, Zanni EE. Genetic mutations affecting human lipoproteins, their receptors, and their enzymes. Ad Hum Genet. 1993;21: 145-319. 1993/01/01. https://doi.org/10.1007/978-1-4615-3010-7_3.

60. Eisenberg DT, Kuzawa CW, Hayes MG. Worldwide allele frequencies of the human apolipoprotein E gene: climate, local adaptations, and evolutionary history. Am J Phys Anthropol. 2010;143:100-11. 2010/08/25. https://doi.org/1 0.1002/ajpa.21298.
61. Heffernan AL, Chidgey C, Peng P, et al. The neurobiology and age-related prevalence of the $\varepsilon 4$ allele of apolipoprotein $\mathrm{E}$ in Alzheimer's disease cohorts. J Mol Neurosci. 2016;60:316-24. 2016/08/09. https://doi.org/10.1 007/s12031-016-0804-x.

\section{Publisher's Note}

Springer Nature remains neutral with regard to jurisdictional claims in published maps and institutional affiliations.
Ready to submit your research? Choose BMC and benefit from:

- fast, convenient online submission

- thorough peer review by experienced researchers in your field

- rapid publication on acceptance

- support for research data, including large and complex data types

- gold Open Access which fosters wider collaboration and increased citations

- maximum visibility for your research: over $100 \mathrm{M}$ website views per year

At BMC, research is always in progress.

Learn more biomedcentral.com/submissions 